\title{
Dalla Letteratura
}

A cura di Remo Melchio, Luca Dutto, Enrica Rinaudo

Dipartimento d'Emergenza Azienda Ospedaliera S. Croce e Carle, Cuneo

\section{La valutazione clinica della sospetta trombosi venosa profonda}

Come per la maggior parte delle patologie, anche per la trombosi venosa profonda (TVP) la valutazione clinica (probabilità a priori) precede ovviamente l'esecuzione di test più raffinati che consentono di confermare o di escludere la malattia, con una ragionevole probabilità. Per esprimere come un dato clinico (o un test) modificano la probabilità di confermare (o di escludere) una malattia si utilizzano il rapporti di verosimiglianza (likelihood ratio, LR): un test con un $L R \geq 2$ è un buon test per confermare, mentre un test con un $L R \leq 0,5$ è un buon test per escludere. In che modo i dati clinici, gli score di rischio (Wells) o il giudizio clinico empirico influenzano la probabilità di individuare la TVP? Gli Autori di questo testo hanno voluto rispondere a questo quesito mediante una revisione sistematica (meta-analisi) degli studi prospettici pubblicati sul tema dal 1966 al gennaio 2005, includendone 51 tra i 3501 potenzialmente rilevanti.

Tra gli elementi clinico-anamnestici considerati isolatamente, si verificavano utili ai fini diagnostici (rule in) soltanto l'anamnesi per neoplasie (LR 2,71) e pregressa TVP (LR 2,25), mentre una recente immobilizzazione (LR 1,98), differenza nel diametro del polpaccio (LR $1,80)$ e recente intervento chirurgico $($ LR 1,76$)$ presentavano valori borderline. Nessun criterio clinico anamnestico era sicuramente utile per escludere la TVP, mentre assenza di edema del polpaccio (LR 0,67) o di differenza del diametro del polpaccio (LR 0,57) presentavano valori borderline.

Lo score di Wells si dimostrava più valido, in quanto in pazienti classificati ad alto rischio presentavano una LR di 5,2, mentre quelli classificati a basso rischio presentavano una LR di 0,25. Anche il giudizio clinico empirico presentava una buona performance per l'alto rischio (LR 5,6) e il basso rischio (LR 0,20), ma con un'attesa ampia eterogenicità tra i vari studi esaminati.

Gli autori concludono che singoli aspetti clinici sono di scarsa utilità nella diagnosi di TVP mentre una valutazione complessiva della probabilità clinica mediante score di Wells è più utile.

Commento: lo studio conferisce una conferma evidence based all'uso dello score di Wells nella pratica clinica.

Goodacre $S$ at al.

Metha-Analysis: The Value of Clinical Assessment in the Diagnosis of Deep Venous Thrombosis.

Annal of Internal Medicine 2005; 143: 129-139.

\section{Controllo della frequenza - cardioversione elettrica nei pazienti con fibrillazione atriale e scompenso cardiaco lieve?}

La gestione della fibrillazione atriale è stata segnata dalla pubblicazione nel 2002 sul New England Journal of Medicine degli studi AFFIRM e RACE che hanno fornito una solida base sperimentale per affermare che il controllo della frequenza cardiaca non è inferiore al controllo del ritmo nel prevenire gli eventi cardiovascolari nei pazienti con fibrillazione atriale persistente.

Gli Autori dello studio RACE hanno recentemente pubblicato questo lavoro il cui scopo è di verificare se l'approccio di controllo della frequenza sia valido anche nei pazienti con fibrillazione atriale e scompenso cardiaco lieve-moderato. La questione non è puramente accademica, in quanto è noto che nei pazienti con scompenso cardiaco il mantenimento del ritmo sinusale si associa a una migliore frazione di eiezione, una maggiore tolleranza allo sforzo e ad un migliore consumo massimale di ossigeno.

Il lavoro (si tratta di un'analisi predefinita di un sottogruppo di pazienti dello studio RACE) prende in considerazione 261 pazienti con scompenso cardiaco lievemoderato (> 90\% in classe NYHA II) randomizzati a controllo della frequenza (con digitale, calcio-antagonisti non diidropiridinici o beta bloccanti) o controllo del ritmo con cardioversione elettrica + trattamento con farmaci antiaritmici (sotalolo, amiodarone o antiaritmici di classe IC). A 2 anni dalla randomizzazione l'end point combinato (mortalità cardiovascolare, ospedalizzazione per scompenso, complicanze tromboemboliche, sanguinamento, impianto di pacemaker, effetti collaterali maggiori da farmaci) è stato raggiunto senza differenze significative da $29(22,3 \%)$ dei 130 pazienti sottoposti a controllo della frequenza e da $32(24,4 \%)$ dei pazienti sottoposti a controllo del ritmo (di cui però soltanto il 36\% manteneva il ritmo sinusale al termine dello studio). Come atteso, nel gruppo sottoposto a controllo della frequenza prevalevano la mortalità cardiovascolare, l'ospedalizzazione per scompenso e il sanguinamento, mentre le complicanze tromboemboliche, gli effetti collaterali da farmaci e l'impianto di pacemaker erano presenti in maggior misura nei pazienti sottoposti a controllo del ritmo. Inoltre, nei pazienti che effettivamente mantenevano il ritmo sinusale al termine dello studio, la mortalità, l'ospedalizzazione per scompenso e il sanguinamento si verificavano meno frequentemente. Gli Autori concludono che nei pazienti con scompenso 
cardiaco lieve-moderato il controllo della frequenza non è inferiore al controllo del ritmo. Tuttavia, se il ritmo sinusale può essere mantenuto, l'outcome può migliorare

Commento: Si tratta del primo studio randomizzato su questo aspetto: tuttavia presenta il limite di una scarsa numerosità del campione per cui gli eventi verificatisi sono scarsi (e l'end point combinato molto "ampio"). Il messaggio che continua a emergere da questo e altri recenti studi sulla fibrillazione atriale è che i benefici ottenuti dal mantenimento "farmacologico" del ritmo sinusale vengono abbattuti dalle complicanze tromboemboliche (recidive asintomatiche di fibrillazione atriale) e dagli effetti collaterali dei farmaci antiaritmici. Tuttavia, se si potesse ridurre l'incidenza dello stroke con l'uso appropriato di anticoagulanti nei pazienti sottoposti a controllo del ritmo (soprattutto se ad alto rischio, come quelli con scompenso cardiaco) o con mezzi più efficaci per prevenire la fibrillazione atriale, l'outcome dei pazienti in ritmo sinusale sarebbe migliore.

\section{Hagens VE et al.}

Rate control versus rhythm control for patients with persistens atrial fibrillation with mild to moderate heart failure: Results from the Rate Control versus Electrical cardioversion (RACE) study.

American Heart Journal 2005; 149: 1106-11.

\section{La morfina nel dolore toracico da sindrome coronarica acuta}

La morfina e.v. è largamente usata nel trattamento del dolore toracico in corso di sindrome coronarica acuta. Le linee guida ACC-AHA per il trattamento dei pazienti con angina instabile o infarto miocardico senza sovraslivellamento del tratto ST (NSTE ACS) ne raccomandano l'uso per i pazienti con dolore toracico che non migliora con la somministrazione di nitrati o con dolore ricorrente (raccomandazione di grado 1C). Ad oggi non sono tuttavia disponibili studi clinici randomizzati che ne abbiano valutato sicurezza ed efficacia. In questo studio osservazionale retrospettivo, gli Autori hanno realizzato un'analisi dei dati contenuti nel registro CRUSADE (57.039 pazienti con NSTE ACS giunti in 443 ospedali statunitensi da gennaio 2001 a giugno 2003) relativi ai pazienti trattati con morfina entro 24 ore dalla presentazione (17003 pazienti). È stato registrato un aumento significativo della mortalità intraospedaliera nei pazienti trattati con morfina rispetto a quelli non trattati; tale aumento di rischio è stato rilevato in tutti i sottogruppi di pazienti (scorporando cioè i dati in base ad età, positività o meno dei marker cardiaci, presenza/assenza di sottoslivellamento del tratto ST, presenza o meno di segni di scompenso congestizio). Gli Autori hanno altresì confrontato i dati relativi al trattamento con nitroglicerina e.v. rispetto al trattamento con morfina sola o con l'associazione di entrambe. Anche in questo caso l'uso della morfina e.v., sola o combinata con nitroglicerina e.v., è risultato essere associato ad un rischio più elevato di mortalità e ad un peggioramento degli outcome clinici (rischio corretto più elevato di infarto miocardico, shock cardiogeno, scompenso cardiaco congestizio), e ciò a dispetto del fatto che l'uso di morfina + nitroglicerina risultasse più spesso condotto in concomitanza con terapie evidence-based.

Commento: I risultati di questa analisi condotta su un campione cospicuo di pazienti possono sollevare dubbi circa l'efficacia e la sicurezza dell'uso della morfina e.v. in corso di sindrome coronarica acuta. Tuttavia, come afferma anche l'editoriale, lo studio suscita più domande che risposte: si tratta di uno studio retrospettivo osservazionale, con tutti i possibili bias di questo approccio; soprattutto non è chiaro se la morfina possa essere stata somministrata non solo a scopo antalgico ma anche per il sollievo della fase agonica di pazienti con NSTE ACS, il che potrebbe spiegare l'associazione con la mortalità. In attesa di un eventuale studio randomizzato che possa dirimere questi dubbi, la morfina continua a rimanere un farmaco cardine nel trattamento del dolore toracico in corso di sindrome coronarica acuta.

Meine T et al.

"Association of intravenous morphine use and outcomes in acute coronary sindrome: results from the CRUSADE Quality Improvement Initiative".

American Heart Journal 2005; 149: 1043-49.

\section{Nebulizzatori o aerosol dosato con spaziatore nell'attacco d'asma?}

L'Autore di questo lavoro si propone di riassumere una revisione sistematica della Cochrane Library del 2003. La revisione si proponeva di confrontare l'uso di broncodilatatori nell'attacco d'asma acuto somministrati mediante nebulizzazione aerosolica con quello di broncodilatatori somministrati mediante aerosol dosati con l'ausilio di spaziatori. Lo studio, pur focalizzando poi l'attenzione sul trattamento dell'attacco asmatico nell'adulto, ha analizzato 22 trial clinici nei dipartimenti di emergenza ed in comunità e 5 trial in pazienti ospedalizzati arrivando a produrre un campione di circa 1076 bambini e 444 adulti. Dal confronto emerge che, in gruppi di pazienti omogenei per parametri fisiologici, la modalità di somministrazione di beta2-agonisti non pare influenzare né il tasso di ricovero né la lunghezza della degenza.

Lo studio ha escluso però le situazioni di male asmatico e di asma life-threatinig, pertanto i risultati vanno applicati sono per gli attacchi di asma lieve e moderata. La revisione giunge alla conclusione che gli aerosol dosati producono gli stessi outcome del broncodilatatore somministrato per via aerosolica nebulizzata. Nel commento degli Autori viene puntualizzato il fatto che la somministrazione di farmaci per nebulizzazione è tuttavia associata ad un incremento dei costi e del rischio di infezione quando si usano aerosolizzatori in comune per più pazienti. L'invito da parte degli Autori pertanto è di incrementare l'uso di aerosol dosati con spaziatore negli attacchi d'asma lieve e moderato (nelle situazioni 
dove la compliance del paziente lo permetta) da parte degli operatori della Medicina d'Emergenza.

B Diner

Nebulizers versus inhalers with spacers for acute asthma.

Annals of Emergency Medicine 2004; 43: 410-412.

\section{Quale strategia per la diagnosi di embolia polmonare?}

Per diagnosticare o escludere un'embolia polmonare (problema che si pone quotidianamente al Medico d'Urgenza) sono disponibili numerosi strumenti validati dalla letteratura: la scintigrafia polmonare perfusionale (studio PIOPED), la TC spirale, l'ecocardiogramma, la risonanza magnetica nucleare, da soli o più spesso associati al D-dimero e all'ecografia compressiva degli arti inferiori. Ma di fronte al singolo paziente, qual è la strategia migliore da utilizzare? Il gruppo di PierreMarie Roy del Dipartimento d'Emergenza di Parigi, per rispondere al quesito, ha realizzato una metanalisi pubblicata su British Medical Journal di luglio, confrontando 48 articoli selezionati da 1012 (3329 pazienti con embolia polmonare su 11.004 sospetti), e ha calcolato i likelihood ratio (LR) dei vari test, fornendo quindi una strategia diagnostica in funzione della probabilità clinica a priori di embolia polmonare.

Nei pazienti a rischio alto o intermedio la TC spirale rappresenta lo strumento migliore per confermare la diagnosi $(\mathrm{LR}+=24,1)$, così come un risultato ad alta probabilità della scintigrafia (LR+ $=18,3$ ) o dell'ecografia compressiva degli arti inferiori $(\mathrm{LR}+=16,2)$. Nei pazienti a rischio basso o intermedio, un D-dimero ELISA $<500 \mu \mathrm{g} / \mathrm{l}$, una scintigrafia normale o quasi normale o una TC spirale negativa associata a un'ecografia compressiva negativa riducono il rischio post test di embolia polmonare a meno del 5\%. L'ecocardiogramma si è mostrato utile soltanto per confermare la diagnosi nei pazienti a rischio alto. Come regola generale, concludono gli Autori, i risultati indicano che quando non vi è accordo tra la probabilità clinica e il risultato del test, è necessario eseguire ulteriori indagini di conferma o di esclusione.

A un analogo risultato sono giunti i radiologi e i clinici del Brigham and Women's Hospital di Boston, che hanno pubblicato su JAMA di aprile un'interessante metanalisi sul ruolo della TC spirale nell'escludere l'embolia polmonare: il lavoro (che ha incluso 15 studi per un totale di 3500 pazienti) è stato condotto con l'obiettivo di verificare la prognosi dei pazienti a cui non era stata iniziata la terapia anticoagulante in seguito al risultato negativo della TC. Grazie a un potere predittivo negativo del 99,1\%, è sicuro, concludono gli Autori, non intraprendere la terapia anticoagulante dopo una TC spirale negativa, nei pazienti a rischio basso-intermedio.

Commento: Si tratta di due importanti metanalisi che confermano il ruolo ormai di primo piano della TC spirale nella gestione del paziente con sospetto di embolia polmonare e rimarcano la necessità della stratifica- zione clinica a priori del rischio per la corretta interpretazione dei risultati dei test.

Quiroz R et al.

Clinical validity of a negative computed tomography scan in patients with suspected pulmonary embolism. A systematic review. JAMA 2005; 293: 2012-17.

Roy Pet al.

Systematic review and meta-analysis of strategies for the diagnosis of suspected pulmonary embolism.

British Medical Journal 2005; 331: 259-263.

\section{BNP è meglio dell'ecocardiogramma nel paziente dispnoico in Pronto Soccorso?}

La dispnea è uno dei sintomi di presentazione al Pronto Soccorso più comuni. Tra i pazienti con dispnea acuta, il B-type Natriuretic Peptide (BNP) si è dimostrato utile per identificare con una discreta accuratezza diagnostica (83\% per un cut off di $100 \mu \mathrm{g} / \mathrm{ml}$, Maisel N Eng J Med 2002) quelli affetti da scompenso cardiaco. Gli Autori di questo studio, pubblicato sul numero di luglio di Chest, hanno voluto confrontare la performance diagnostica del BNP con la valutazione ecocardiografica della funzione sistolica del ventricolo sinistro nell'ambiente del Pronto Soccorso, utilizzando i dati dello studio multicentrico BNP Multinational Study (1586 pazienti di cui 709 sottoposti a ecocardiografia). I risultati sono particolarmente interessanti in quanto dimostrano per il BNP una sensibilità (cut off $>100 \mu \mathrm{g} / \mathrm{ml}$ ) dell' $89 \%$ con una specificità del $73 \%$ versus una sensibilità dell'ecocardiogramma $(\mathrm{EF}<50 \%)$ del $70 \%$ e una specificità del $77 \%$. Vi è una differenza significativa anche tra le curve ROC (una stima dell'accuratezza diagnostica complessiva a diversi livelli di cut off) delle due metodiche. Inoltre entrambe si mostrano predittori indipendenti di scompenso all'analisi multivariata.

Gli Autori concludono che il BNP è superiore all'ecocardiogramma bidimensionale nell'identificare lo scompenso cardiaco e che la combinazione delle due metodiche ha un marcato valore diagnostico additivo.

Commento: Il lavoro riveste un particolare interesse nel definire il ruolo del BNP in urgenza. Tuttavia, bisogna sottolineare come il confronto con l'ecocardiogramma prenda in considerazione soltanto la frazione di eiezione che, tra i parametri che vengono forniti dalla metodi$\mathrm{ca}$, è utile per identificare la disfunzione sistolica come causa di scompenso. I parametri che esprimono la disfunzione diastolica o valvolare, spesso non misurati nel contesto di un esame di Pronto Soccorso, ma utili per una caratterizzazione migliore della performance cardiaca, non vengono considerati.

Steg PG et al.

B-tipe natriuretic peptide and echocardiographic determination of ejection fraction in the diagnosis of congestive heart failure in patients with acute dispnea.

Chest 2005; 128: 21-29. 